\title{
Secretion of proinflammatory cytokines by normal human melanocytes in response to lipopolysaccharide
}

\author{
Irena Tam ${ }^{\bowtie}$ and Krystyna Stępień \\ Department of Instrumental Analysis, Medical University of Silesia, Sosnowiec, Poland
}

\begin{abstract}
A large body of evidence suggests that epidermal melanocytes are an integral part of the skin immune system and can be considered immunocompetent cells. Recently, it has been reported that human melanocytes constitutively express Toll-like receptors and may be involved in the induction of several inflammatory cytokines. In the study the secretion of IL-1 1, IL- 6 and TNF- $\alpha$ by cultured normal melanocytes was investigated after stimulation with lipopolysaccharide. LPS increased the secretion of IL-1 $\beta$ in a dose-dependent manner. IL$1 \beta$ stimulated release of IL- 6 and TNF- $\alpha$ by melanocytes, whereas LPS activated production of TNF- $a$, but not of IL-6. These observations indicate that LPS can participate in the regulation of cytokine activity in normal human melanocytes and suggest that cytokines released by melanocytes could affect melanocytes themselves or/and other cells of the epidermis.
\end{abstract}

Keywords: melanocytes, proinflammatory cytokines, lipopolysaccharide

Received: 03 March, 2011; revised: 25 October, 2011; accepted: 28 November, 2011; available on-line: 30 November, 2011

\section{INTRODUCTION}

Human melanocytes are unique secretory epidermal cells capable of producing a multifunctional pigment melanin. Production of melanin is regulated by various factors via receptor-dependent and -independent pathways, in a hormonal, auto-, para-, or intracrine manner (Slominski et al., 2004a). The most important regulators of melanogenesis are melanocortin-1 receptor (MC1R) and its ligands $\alpha$-melanocyte-stimulating hormone $(\alpha-\mathrm{MSH})$ and adrenocorticotropic hormone (ACTH), that are produced by the enzymatic cleavage of proopiomelanocortin (POMC) in melanocytes, keratinocytes and other skin cell types. Binding of $\alpha-\mathrm{MSH}$ or ACTH to MC1R on melanocytes stimulates the expression of the melanogenic cascade and eumelanin synthesis (Slominski et al., 2004b). The pigment, synthesized within melanocytes and then transferred to surrounding keratinocytes, absorbs ultraviolet light and its primary role is to protect skin from solar radiation. Melanin can also act as a scavenger of free radicals, effective metal ions chelator and antioxidant (Herrling et al., 2008; Stępień, 2010). In addition to the photoprotective function, melanocytes also exhibit neuroendocrine activity and produce classical stress neurotransmitters, neuropeptides and hormones (Slominski, 2009).

A large body of evidence suggests that epidermal melanocytes are an integral part of the skin immune system (SIS) and can be considered immunocompetent cells (Tam \& Stępień, 2007). These melanin-producing cells express major histocompatibility complex class II molecules and intercellular adhesion molecules (ICAM, VCAM and CD40), and may be capable of antigen processing and presentation (Smit et al., 1993; Lu et al., 2002). Melanocytes are able to phagocytose and the melanin pigment itself can act as a physical barrier against microorganisms (Mackintosh, 2001). Moreover, reactive quinone intermediates and hydrogen peroxide generated during melanin synthesis exert strong antimicrobial activity (Plonka et al., 2009). Recently, it has been reported that human melanocytes constitutively express Toll-like receptors 2-5, 7, 9 and 10 at the protein level (Ahn et al., 2008a; Yu et al., 2009; Jin \& Kang, 2010). In human, TLRs recognize constituents of microbial cell walls or pathogen-specific nucleic acids, trigger NF- $x \mathrm{~B}$ (nuclear factor kappa light chain enhancer of activated B cells) and/or MAPK (mitogen-activated protein kinase) signalling pathway, and might be involved in the induction of several inflammatory cytokines and chemokines (Ahn et al., 2008b; Yu et al., 2009; Jin \& Kang, 2010). Furthermore, TLR-mediated activation is very similar to that of another essential cytokine receptor, inteleukin-1 receptor (IL-1R). Both TLRs and IL-1R share the MyD88 adaptor molecule and promote the production of proinflammatory cytokines (Verstrepen et al., 2008).

Melanocytes could also act as regulators of the skin's immune response by producing and releasing several immunosuppressive molecules including POMC-derived ACTH and $\alpha$-MSH (Slominski et al., 2000), cortisol, corticosterone and other steroids (Slominski et al., 1999; 2004b; 2005). In particular, $\alpha-\mathrm{MSH}$ has a powerful antiinflammatory potential and affects various pathways implicated in the regulation of inflammation (Brzoska et al., 2008). Furthermore, intermediates of melanogenesis, especially L-DOPA (L-3,4-dihydroxyphenylalanine) and/ or products of its oxidation can act as potent immunosuppressors. It has been demonstrated that L-DOPA inhibits lymphocyte proliferation and abolishes production of proinflammatory cytokines by activated lymphocytes (Slominski \& Goodman-Snitkoff, 1992; Slominski et al., 2009).

Most of the data on immunocompetence of human melanocytes have emerged from studies on melanoma (Mattei et al., 1994; Lázár-Molnár et al., 2000; Kozłowska

email: tami@sum.edu.pl

Abbreviations: ACTH, adrenocorticotropic hormone; ELISA, enzyme-linked immunosorbent assay; IFN, interferon; IL, interleukin; $\mathrm{LDH}$, lactate dehydrogenase; L-DOPA, L-3,4-dihydroxyphenylalanine; LPS, lipopolysaccharide; $\mathrm{MSH}$, melanocyte-stimulating hormone; POMC, proopiomelanocortin; PCR, polymerase chain reaction; TNF, tumour necrosis factor; TLRs, Toll-like receptors. 
et al., 2001; Kholmanskikh et al., 2010; Elias et al., 2010) and little is known about the cytokine-producing potential of normal melanocytes and their role in the network of cytokine interactions. The aim of the present study was to examine the ability of normal human melanocytes to secrete IL-1 $\beta$, IL- 6 and TNF- $\alpha$, the most important cytokines associated with skin inflammation, in response to bacterial LPS. Additionally, we investigated the potential of IL-1 $\beta$ to induce TNF- $\alpha$ and IL- 6 release in melanocyte cultures.

\section{MATERIALS AND METHODS}

Materials. Primary human epidermal melanocytes from neonatal foreskin of moderately pigmented donor (HEMn-MP) were purchased from Cascade Biologics/ GIBCO Invitrogen. Melanocyte cultures were grown in melanocyte growth medium, consisting of Medium 254, human melanocyte growth supplement (HMGS) and antibiotics (Gentamicin \& Amphotericin B solution) (Cascade Biologics/GIBCO Invitrogen). Trypsin neutralizer and $0.025 \%$ trypsin/EDTA solutions were from GIBCO Invitrogen. Dulbecco's PBS buffer was obtained from PAA Cell Culture Company. Lipopolysaccharide from Escherichia coli (026:B6) was purchased from Sigma-Aldrich, Inc. Recombinant human IL-1 $\beta$ protein and Quantikine Human TNF- $\alpha$, IL- 6 and IL-1 $\beta$ Immunoassays were from R\&D Systems (Minneapolis, USA). CytoTox96 ${ }^{\circledR}$ Non-Radioactive Cytotoxicity Assay and CellTiter-Glo Luminescent Cell Viability Assay were obtained from Promega Corporation.

Melanocyte culture. Human epidermal melanocytes were cultured according to the supplier's recommendations. Briefly, cryopreserved primary melanocytes were transferred into $25-\mathrm{cm}^{2}$ tissue culture flasks at a density of $5 \times 10^{3}$ cells $/ \mathrm{cm}^{2}$ and were grown in melanocyte growth medium in a humidified incubator with $5 \%$ $\mathrm{CO}_{2} / 95 \%$ air at $37^{\circ} \mathrm{C}$. The culture medium was changed every other day. After cells became about $80 \%$ confluent the medium was removed and melanocytes were subcultured using conventional trypsinization method with trypsin/EDTA and trypsin neutralizer solutions.

Melanocyte stimulation. For stimulation of cytokine production, melanocytes from the 4th and 5th passages were used. The cells resuspended in supplemented Medium 254 were plated into 96-well microplates at a density of $10^{4}$ cells / well. After two days of incubation, the media were refreshed and the incubation continued for an additional 24 hours. Then, the media were replaced with fresh media containing various concentrations of LPS $(0.01-20 \mu \mathrm{g} / \mathrm{ml})$ or IL-1 $\beta(0.01-10 \mathrm{ng} / \mathrm{ml})$. Melanocytes cultured without the stimulants were used as a control. After a 24-h stimulation culture supernatants were collected, and intracellular adenosine triphosphate (ATP) levels were measured in melanocytes. The supernatant samples were stored at $-70^{\circ} \mathrm{C}$ until assayed for cytokine and lactate dehydrogenase $(\mathrm{LDH})$ activity.

Estimation of cell viability. The parameters used for evaluating melanocyte viability in the stimuli-treated and non-treated cultures were LDH leakage and intracellular ATP level.

$\mathrm{LDH}$ release into the culture medium is an indicator of cell death resulting in cell membrane damage. The amount of $\mathrm{LDH}$ in the medium was determined using the CytoTox $96^{\circledR}$ Non-Radioactive Cytotoxicity Kit, according to the manufacturer's instruction. The absorbance of red formazan product was recorded at $490 \mathrm{~nm}$ using a TRIAD LT microplate reader (Dynex Technolo-

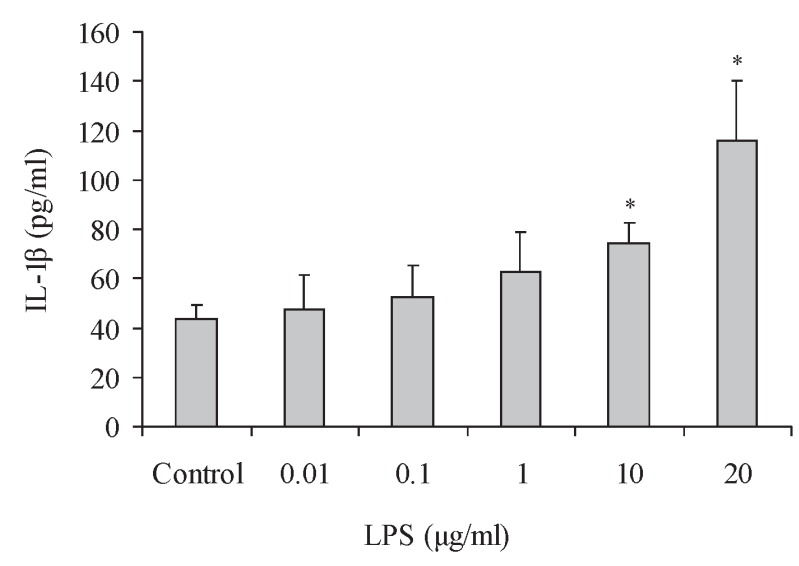

Figure 1. Effect of LPS on IL-1 $\beta$ secretion by normal human epidermal melanocytes.

Culture supernatants were collected 24 hours after LPS stimulation and IL-1 $\beta$ concentrations were determined by ELISA. The values indicate the mean \pm S.D. $(n=6)$. ${ }^{*} P<0.05$ vs control.

gies, USA). The cytotoxicity of the tested compounds was calculated according to the formula: \% cytotoxicity $=$ (stimulated cells $\mathrm{LDH}$ release - spontaneous $\mathrm{LDH}$ release/maximum $\mathrm{LDH}$ release — spontaneous $\mathrm{LDH}$ release $\times 100$. Maximum $\mathrm{LDH}$ release was obtained after lysis of the cells with Lysis Solution provided by the producer.

The presence of metabolically active melanocytes in the cultures was estimated by measurement of the cellular ATP level. A bioluminescence assay based on the detection of ATP via luciferase - catalyzed reaction was performed in accordance with the manufacturer's protocol (CellTiter-Glo Luminescent Cell Viability Assay). The luminescent signal, proportional to the amount of ATP, was recorded on a TRIAD LT microplate reader.

Cytokine measurements. The cytokine contents in cell-free culture supernatants were determined with commercial enzyme-linked immunosorbent assay (ELISA) kits, according the manufacturer's directions (Quantikine). The limits of detection were: $4.4 \mathrm{pg} / \mathrm{ml}$ for TNF- $\alpha$, $0.7 \mathrm{pg} / \mathrm{ml}$ for IL-6 and $1 \mathrm{pg} / \mathrm{ml}$ for IL-1 $\beta$. Absorbance was read at $450 \mathrm{~nm}$ using a TRIAD LT microplate reader. The quantification was made on the basis of calibration curves prepared for the cytokines analyzed.

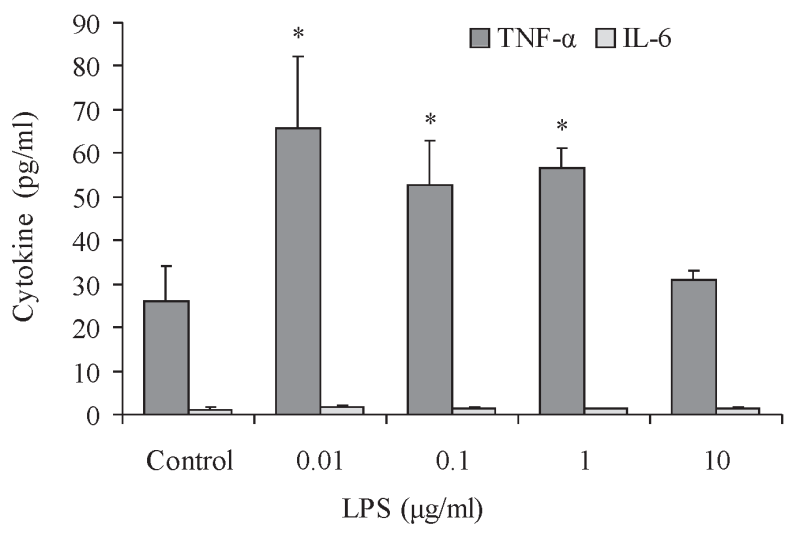

Figure 2. Effect of LPS on IL-6 and TNF-a secretion by normal human epidermal melanocytes.

Culture supernatants were collected 24 hours after LPS stimulation and IL- 6 and TNF- $a$ concentrations were determined by ELISA. The values indicate the mean \pm S.D. $(n=6) .{ }^{*} P<0.05$ vs control. 


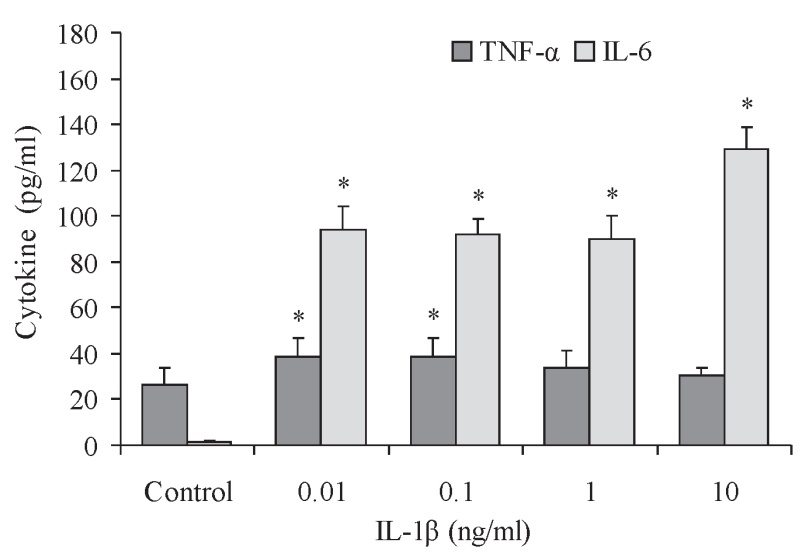

Figure 3. Effect of interleukin $1 \beta$ on IL- 6 and TNF- $\alpha$ secretion by normal human epidermal melanocytes.

Culture supernatants were collected 24 hours after IL-1 $\beta$ stimulation and IL- 6 and TNF- $a$ concentrations were determined by ELISA. The values indicate the mean \pm S.D. $(n=6)$. ${ }^{*} P<0.05$ vs control.

Statistical analysis. Data represent the mean \pm standard deviation (S.D.) from six independent experiments. Comparisons between groups were made by a one-way analysis of variance with post-hoc Tukey's test using Statistica version 8 software (StatSoft Inc., USA). $P$ values less than 0.05 were considered significant.

\section{RESULTS}

\section{Evaluation of melanocyte viability following exposure to LPS or IL-1 $\beta$}

Effects of LPS and IL-1 $\beta$ on melanocyte viability were evaluated on the basis of intracellular ATP levels and $\mathrm{LDH}$ release after 24-h exposition to the stimuli. LPS or IL-1 $\beta$ did not display any significant cytotoxic effect on melanocytes. LDH levels in media were unaffected following exposure to these stimuli. Also, ATP content assay revealed that the viability of melanocytes exposed to LPS and IL-1 $\beta$ in the concentration range tested was comparable to that of cells incubated with medium only.

\section{Modulation of cytokine secretion by LPS or IL-1 $\beta$}

We observed differences in the constitutive production of the cytokines analyzed by melanocytes. IL-1 $\beta$ and TNF- $\alpha$ were detected in the cell supernatants at concentrations of $43.2 \pm 6 \mathrm{pg} / \mathrm{ml}$ and $26.1 \pm 8 \mathrm{pg} / \mathrm{ml}$, respectively, while the constitutive secretion of IL-6 was minimal $(1.2 \pm 0.5 \mathrm{pg} / \mathrm{ml})$.

Stimulation of melanocytes with LPS induced dosedependent increase in IL-1 $\beta$ secretion (Fig. 1). At the highest dose of LPS $(20 \mu \mathrm{g} / \mathrm{ml})$ the secretion was approximately $270 \%$ of control (i.e., $115.8 \pm 24 \mathrm{pg} / \mathrm{ml}$ ). The effect of LPS and exogenous IL-1 $\beta$ on TNF- $\alpha$ and IL-6 production is presented in Figs. 2 and 3. Incubation of melanocytes with increasing concentrations of LPS had no effect in the production of IL-6. In contrast, stimulation of melanocytes with IL-1 $\beta$ resulted in a significant rise in IL-6 secretion (approximately 75 -fold at $0.01-1 \mathrm{ng} / \mathrm{ml}$ and 110 -fold at $10 \mathrm{ng} / \mathrm{ml}$, compared with the control). The both stimuli showed a similar $\mathrm{TNF}-\alpha$-secretion profile. However, in response to LPS the TNF- $\alpha$ secretion increased 2.5 -fold while in response to IL-1 $\beta$ only 1.5 -fold. In the both cases, the largest re- sponse was observed when the stimulus was applied at low doses.

\section{DISCUSSION}

Reports on the production of proinflammatory cytokines by normal human melanocytes are scarce, and the described results are contradictory. Smit et al. (1993) demonstrated by immunohistochemistry that human melanocytes constitutively synthesized IL-1 $\beta$, IL-6 and IL-2. Swope et al. (1994) found melanocytes able to produce IL- $1 \alpha$ and IL-1 $\beta$ using immunocytochemical staining and the PCR technique. In studies by Mattei et al. (1994) cultures of normal melanocytes were positive for RNA transcripts for IL-6 and its receptor (IL-6R), IL-8, IL10 , TNF- $\beta$ and TNFR (TNF receptor), but negative for IL- $1 \alpha$, IL-1 $\beta$ and TNF- $\alpha$. In our study, the quantitation of cytokine production at the protein level was based on immunoenzymatic assay (ELISA). The obtained results showed that IL-1 $\beta$, TNF- $\alpha$ and IL- 6 were constitutively secreted by melanocytes, but the amount of IL-6 released was approximately 40 - and 20 -fold lower than the amount of IL- 1 and $\mathrm{TNF}-\alpha$, respectively.

LPS is a well-known endotoxin which elicits a variety of inflammatory responses. In humans, LPS binds the CD14/TLR4/MD2 receptor complex, which triggers the signaling cascade for many cell types to secrete proinflammatory cytokines and nitric oxide (Hari et al., 2010). LPS-treated melanocytes were found to respond to the endotoxin by activation of TLR4 and TLR2, expression of adaptor molecules CD14 and MyD88, promotion of $\mathrm{NF}-x \mathrm{~B}$ subunit p65 translocation to the nucleus (Ahn et al., 2008a; Yu et al., 2009; Jin \& Kang, 2010) and enhanced expression of some proinflammatory cytokines and chemokines (IL-6, IL-8, CCL2, CCL3 and CCL5) (Yu et al., 2009). We examined changes in the secretion of IL-1 $\beta$, IL- 6 and TNF- $\alpha$ by cultured human melanocytes in response to LPS. Additionally, the potential of IL-1 $\beta$ to induce TNF- $\alpha$ and IL-6 release in melanocyte cultures was assessed. Our results showed that LPS triggered the secretion of IL-1 $\beta$ and TNF- $\alpha$ by normal epidermal melanocytes. Moreover, we observed differences in TNF- $\alpha$ and IL-6 secretion by melanocytes after exposure to LPS and IL-1 $\beta$. The incubation of melanocytes with increasing concentrations of LPS did not affect the production of IL- 6 , while stimulation with IL-1 $\beta$ resulted in a significant growth of IL-6 secretion. These differences may be due to the fact that fewer than ten ligandoccupied IL-1RI receptors are required per cell to induce a strong response and this potency is in contrast to the Toll-like receptor system, which requires a 10 - to 100 fold higher receptor occupancy (Dayer, 2002). Besides, secretion of IL-1, IL- 6 and TNF- $\alpha$ need not always occur in parallel (Burbach et al., 2001) and the cytokines are capable of cross-regulating one another (Radtke et al., 2010). In this context, IL-1 and IL-6 released by activated melanocytes could form a positive feedback loop in upregulating TNF- $\alpha$ and possibly others cytokines of inflammation (Van Der Meer et al., 2005).

It has been reported that IL-1, IL- 6 and TNF- $\alpha$ inhibit human melanocyte proliferation and melanogenesis, and $\mathrm{TNF}-\alpha$ induces apoptosis of these cells (Swope et al., 1991; Shang et al., 2002). On the other hand, recent studies have demonstrated that LPS increases melanin synthesis in human melanocytes and induces pigmentation of cultured skin (Ahn et al., 2008b; Jin \& Kang, 2010). Other mediators of inflammation, such as nitric oxide, histamine and eicosanoids, have also been shown 
to stimulate melanogenesis (Romero-Graillet et al., 1997; Sasaki et al., 2000; Slominski et al., 2004a). Furthermore, postinflammatory hyperpigmentation of the skin is frequently observed in clinical practice (Pandya \& Guevara, 2000; Brajac et al., 2009). These date indicate that epidermal melanocytes can modulate their pigmentation in response to inflammatory conditions. As intermediates of melanogenesis and melanin itself are able to inhibit activity of immune cells (Slominski \& Goodman-Snitkoff, 1992; Mohagheghpour et al., 2000; Slominski et al., 2009), the process of melanogenesis could have immunomodulatory functions. Interestingly, inhibition of melanogenesis has been proposed as an adjuvant strategy in the treatment of melanotic melanomas (Slominski et al. 1998; 2009).

Overproduction of IL-6, TNF- $\alpha$ and IL-1 in the skin is considered a mediator of transition from acute to chronic inflammation and can be implicated in the development of some melanotic disorders (Imokawa, 2006). It is hypothesized that aberrant production of cytokines by melanocytes may be associated with pigmentary changes (Abdel-Malek \& Kadekaro, 2006; Hasegawa et al., 2008; Jin \& Kang, 2010) and play an important role in the pathogenesis of human diseases, such as lichen planus, psoriasis, vitiligo and melanoma (Clark, 2001; Moretti et al., 2002; Brajac et al., 2009; Hari et al., 2010).

\section{Acknowledgements}

This study was supported by grants of the Medical University of Silesia (KNW-2-131/09 and KNW-1$115 / 10)$.

\section{REFERENCES}

Abdel-Malek Z, Kadekaro A (2006) Human pigmentation: its regulation by ultraviolet light and by endocrine, paracrine, and autocrine factors. In The Pigmentary System: Physiology and Pathophysiology - 2nd edn. Nordlund J, Boissy R, Hearing V, King R, Oetting W, Ortonne J, eds, pp 410-420. Blackwell Publishing Ltd, Oxford, UK.

Ahn J, Park T, Jin S, Kang H (2008a) Human melanocytes express functional Toll-like receptor 4. Exp Dermatol 17: 412-417.

Ahn J, Jin S, Kang H (2008b) LPS induces melanogenesis through p38 MAPK activation in human melanocytes. Arch Dermatol Res 300: 325-329.

Brajac I, Kaštelan M, Prpič-Massari L, Periša D, Lončarek K, Malnar D (2009) Melanocyte as a possible key cell in the pathogenesis of psoriasis vulgaris. Med Hypotheses 73: 254-256.

Brzoska T, Luger T, Maaser C, Abels C, Bohm M (2008) $\alpha$-Melanocytestimulating hormone and related tripeptides: biochemistry, antiinflammatory and protective effects in vitro and in vivo, and future perspectives for the treatment of immune-mediated inflammatory diseases. Endocr Rev 29: 581-602.

Burbach G, Ansel J, Amstrong Ch (2001) Cytokines in the skin. In The Biology of the Skin. Freinkel R, Woodley D, eds, pp 299-332. The Parthenon Publishing, New York, USA.

Clark J (2001) Role of cytokines in pathology of melanoma and use of biologic response modifiers in melanoma. In Methods in Molecular Medicine, Melanoma Techniques and Protocols: Molecular Diagnosis, Treatment, and Monitoring. Nickoloff B, Hood L, eds, pp 241-267. Humana Press, New Jersey, USA.

Dayer J (2002) Evidence for the biological modulation of IL-1 activity: the role of IL-1Ra. Clin Exp Rheumatol 20: S14-S20.

Elias E, Hasskamp J, Sharma B (2010) Cytokines and growth factors expressed by human cutaneous melanoma. Cancers 2: 794-808.

Hari A, Flach T, Shi Y, Mydlarski R (2010) Toll-like receptors: role in dermatological disease. Mediators Inflamm 2010: 1-16.

Hasegawa J, Goto Y, Murata H, Takata M, Saida T, Imokawa G (2008) Downregulated melanogenic paracrine cytokine linkages in hypopigmented palmoplantar skin. Pigment Cell Melanoma Res 21: 687-699.

Herrling T, Jung K, Fuchs J (2008) The role of melanin as protector against free radicals in skin and its role as free radical indicator in hair. Spectrochim Acta Part A 69: 1429-1435.

Imokawa G (2006) Paracrine interactions of melanocytes in pigmentary disorders. In The Pigmentary System: Physiology and Pathophysiology - 2nd edn. Nordlund J, Boissy R, Hearing V, King R, Oetting W, Ortonne J, eds, pp 421-444. Blackwell Publishing Ltd, Oxford, UK.

Jin $H$, Kang $H$ (2010) Activation of toll-like receptors 1, 2, 4, 5, and 7 on human melanocytes modulate pigmentation. Ann Dermatol 22: 486-489.

Kholmanskikh O, van Baren N, Brasseur F, Ottaviani S, Vanacker J, Arts N, van der Bruggen P, Coulie P, De Plaen E (2010) Interleukins $1 \alpha$ and $1 \beta$ secreted by some melanoma cell lines strongly reduce expression of $\mathrm{MITF}-\mathrm{M}$ and melanocytes differentiation antigens. Int J Cancer 127: 1625-1636.

Kozłowska K, Zarzeczna M, Cichorek M (2001) Characterization of $\mathrm{NO}$ and cytokine release by transplantable melanoma cell lines in relationship to their differentiation. Arch Immunol Ther Exp 49: S103-S109.

Lázár-Molnár E, Hegyesi H, Tóth S, Falus A (2000) Autocrine and paracrine regulation by cytokines and growth factors in melanoma. Cytokine 12: 547-554.

Lu Y, Zhu W, Tan C, Yu G, Gu J (2002) Melanocytes are potential immunocompetent cells: evidence from recognition of immunological characteristics of cultured human melanocytes. Pigment Cell Res 15: $454-460$.

Mackintosh J (2001) The antimicrobial properties of melanocytes, melanosomes and melanin and the evolution of black skin. $J$ Theor Biol 211: 101-113.

Mattei S, Colombo M, Melani C, Silvani A, Parmiani G, Herlyn M (1994) Expression of cytokine/growth factors and their receptors in human melanoma and melanocytes. Int J Cancer 56: 853-857.

Mohagheghpour N, Waleh N, Garger S, Dousman L, Grill L, Tusé D (2000) Synthetic melanin suppresses production of proinflammatory cytokines. Cell Immunol 199: 25-36.

Moretti S, Spallanzani A, Amato L, Hautmann G, Gallerani I, Fabiani M, Fabbri P (2002) New insights into the pathogenesis of vitiligo: imbalance of epidermal cytokines at sites of lesions. Pigment Cell Res 15: 87-92.

Pandya A, Guevara I (2000) Disorders of hyperpigmentation. Dermatol Clin 18: 91-98.

Plonka P, Passeron T, Brenner M, Tobin D, Shibahara S, Thomas A, Slominski A, Kadekaro A, Hershkovitz D, Peters E, Nordlund J, Abdel-Malek Z, Takeda K, Paus R, Ortonne J, Hearing V, Schallreuter K (2009) What are melanocytes really doing all day long...?. Exp Dermatol 18: 799-819.

Radtke S, Wüller S, Yang X, Lippok B, Mütze B, Mais C, Schmitz-Van de Leur H, Bode J, Gaestel M, Heinrich P, Behrmann I, Schaper F, Hermanns H (2010) Cross-regulation of cytokine signalling proinflammatory cytokines restrict IL-6 signalling through receptor internalisation and degradation. I Cell Sci 123: 947-959.

Romero-Graillet C, Aberdam E, Clement M, Ortonne J, Ballotti R (1997) Nitric oxide produced by ultraviolet irradiated keratinocytes stimulates melanogenesis. I Clin Invest 99: 635-642.

Sasaki M, Horikoshi T, Uchiwa H, Miyachi Y (2000) Up-regulation of tyrosinase gene by nitric oxide in human melanocytes. Pigment Cell Res 13: 248-252.

Shang J, Eberle J, Geilen C, Hossini A, Fecker L, Orfanos C, Tebbe $\mathrm{B}$ (2002) The role of nuclear factor-kappaB and melanogenesis in tumor necrosis factor-alpha-induced apoptosis of normal human melanocytes. Skin Pharmacol Appl Skin Physiol 15: 321-329.

Slominski A (2009) Neuroendocrine activity of the melanocytes. Exp Dermatol 18: 760-763.

Slominski A, Goodman-Snitkoff G (1992) Dopa inhibits induced proliferative activity of murine and human lymphocytes. Anticancer Res 12: $753-756$.

Slominski A, Paus R, Mihm M (1998) Inhibition of melanogenesis as an adjuvant strategy in the treatment of melanotic melanomas: selective review and hypothesis. Anticancer Res 18: 3709-3716.

Slominski A, Gomez-Sanchez C, Foecking M, Wortsman J (1999) Metabolism of progesterone to DOC, corticosterone and 18OHDOC in cultured human melanoma cell. FEBS Lett 445: 364-366.

Slominski A, Wortsman J, Luger T, Paus R, Solomon S (2000) Corticotropin releasing hormone and proopiomelanocortin involvement in the cutaneous response to stress. Physiol Rev 80: 979-1020.

Slominski A, Tobin D, Shibahara S, Wortsman J (2004a) Melanin pigmentation in mammalian skin and its hormonal regulation. Physiol Rev 84: 1155-1228.

Slominski A, Zjawiony J, Wortsman J, Semak I, Stewart J, Pisarchik A, Sweatman T, Marcos J, Dunbar C, Tuckey R (2004b) A novel pathway for sequential transformation of 7-dehydrocholesterol and expression of the P450scc system in mammalian skin. Eur J Biochem 271: 4178-4188.

Slominski A, Zbytek B, Szczesniewski A, Semak I, Kaminski J, Sweatman T, Wortsman J (2005) CRH stimulation of corticosteroids production in melanocytes is mediated by ACTH. Am J Physiol Endocrinol Metab 288: E701-E706.

Slominski A, Zbytek B, Slominski R (2009) Inhibitors of melanogenesis increase toxicity of cyclophosphamide and lymphocytes against melanoma cells. Int J Cancer 124: 1470-1477. 
Smit N, Le Poole I, Wijngaard R van den, Tigges A, Westerhof W, Das P (1993) Expression of different immunological markers by cultured human melanocytes. Arch Dermatol Res 285: 356-365.

Stępień K (2010) The role of melanocytes in protection against photooxidative stress. Post Bioch 56: 290-297.

Swope V, Abdel-Malek Z, Kassem L, Nordlund J (1991) Interleukins 1 $\alpha$ and 6 and tumor necrosis factor $-\alpha$ are paracrine inhibitors of human melanocytes proliferation and melanogenesis. I Invest Dermatol 96: $180-185$.

Swope V, Sauder D, McKenzie R, Sramkoski R, Krug K, Babcock G, Nordlund J, Abdel-Malek Z (1994) Synthesis of interleukin- $1 \alpha$ and $\beta$ by normal human melanocytes. J Invest Dermatol 102: 749-753.
Tam I, Stępień K (2007) Melanocytes — immunocompetent pigmented cells. Post Dermatol Alergol 24: 188-193.

Van Der Meer J, Helle M, Aerden L (2005) Comparison of the effects of recombinant interleukin 6 and recombinant interleukin 1 on nonspecific resistance to infection. Eur J Immunol 19: 413-416.

Verstrepen L, Bekaert T, Chau T, Tavernier J, Chariot A, Beyaert R (2008) TLR-4, IL-1R and TNF-R signaling to NF- $x$ B: variations on a common theme. Cell Mol Life Sci 65: 2964-2978.

Yu N, Zhang S, Zuo F, Kang K, Guan M, Xiang L (2009) Cultured human melanocytes express functional toll-like receptors $2-4,7$ and 9. J Dermatol Sci 56: 113-120. 